\title{
FOREWORD
}

\section{An Appreciation of Professor A. Busemann}

Dr. Adolf Busemann, born in Lubeck, Germany, in 1901, is a Professor Emeritus of Aerospace Engineering Sciences at the University of Colorado, Boulder, Colorado. He received his Doctorate in Engineering in 1924 and worked with Ludwig Prandtl at the Kaiser Wilhelm Institute, now the Max Planck Institute. He is a Member of the Max Planck Association for the Advancement of Science and of the Academy

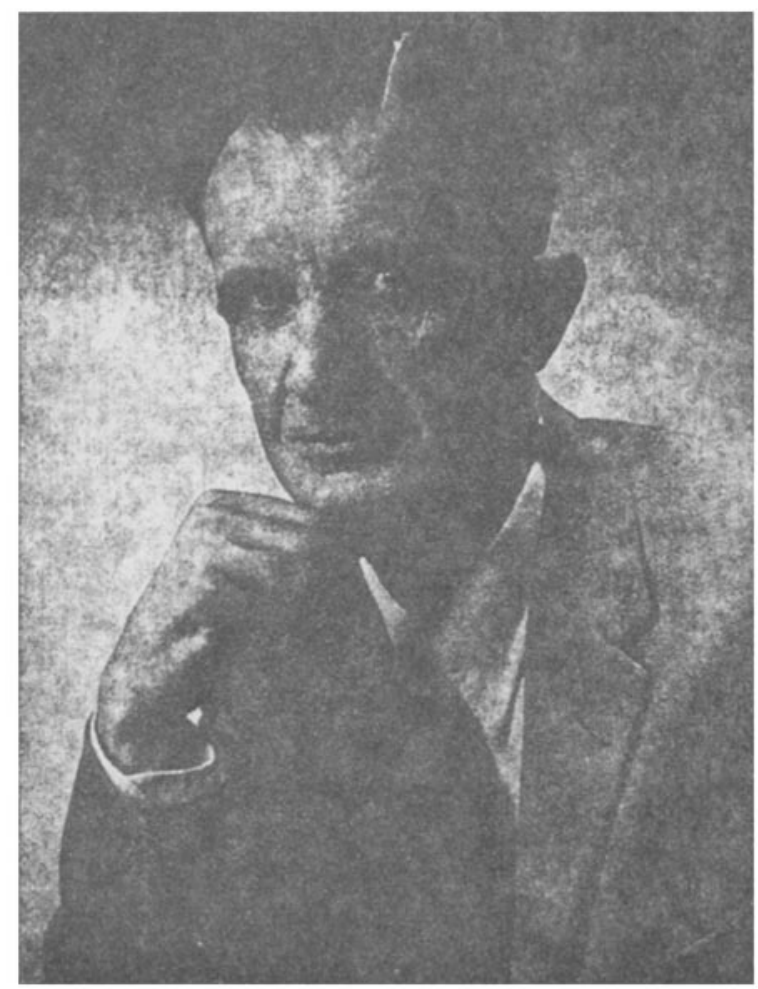

Professor Adolf Busemann

(C) 1975 Plenum Publishing Corporation, 227 West 17th Street, New York, Y. Y. 10011. No part of this publication may be reproduced, stored in a retrieval system, or transmitted, in any form or by any means, electronic, mechandcal, photocopying, microflining, recording, or otherwise, without written permission of the publisher. 
of Sciences of Turin, Turin, Italy. He is a Fellow of the American Institute of Aeronautics and Astronautics and is a Member of the International Academy of Astronautics, headquartered in Paris, France.

At the Volta Congress of 1935 in Rome, he proposed the use of the swept-back wing for high-speed aircraft, a wing which is used on all high-speed military and commercial planes today. Dr. Busemann has done pioneering work in all areas of aerodynamics, and many of his discoveries have passed the test of time and are now essential features in standard textbooks on gasdynamics.

Over the years, Dr. Busemann has received numerous awards for his many and long-lasting contributions to the advancement of science. In 1944, he was awarded the Carl Bosch Award for Advancement in Aeronautical Sciences. His work in plasma physics led to a patent for the design of a plasma accelerator for which he was given a special NASA cash award. In 1967, he received the AIAA Sylvanus Albert Reed Award for pioneering work in supersonic wing theory and for his early contributions to sonic bocm theory. He is considered to be the father of supersonic aerodynamics and received the honorary degree of Doctor of Natural Sciences at Aachen, Germany, in 1966. In 1970, Dr. Busemann was elected to the National Academy of Engineering in recognition of his pioneering discoveries in high-speed aerodynamics and supersonic flight, especially the swept-back wing and his studies of conical flows, and many contributions to engineering education.

Dr. Busemann's acquaintance with optimization theory started as an engineering student and expanded by listening to Prandtl's lectures. The large variety of relevant physical problems and their solutions, partly observable in nature and partly discovered as advances in engineering, was very enlightening for Busemann. There is, for instance, in electrostatics, the automatic minimum energy distribution for charges on a conductor of any shape. This simple solution may serve as a first step toward the classical two-dimensional optimum distribution of vortex pairs for a given lift derived by Max Munk (or even of vortex quadripoles for a given moment derived by Albert Betz), within the trailing vortex sheet behind a wing of given span. With this eye-opener, Busemann was able to find the vortex distribution of minimum energy for several new cases (Ref. 1). This experience became extremely valuable when Theodore Von Kármán, at the Volta Meeting of 1935 in Rome, presented the analogy between the mutual energy of vortex pairs in a sheet and the one-sided interference drag between point sources in three-dimensional space, arranged on a line in the flow 
direction at supersonic speeds. The consequences were illustrated by Von Kármán's ogive as the optimum tip shape of given length on a cylindrical afterbody. Not only was Busemann ready to visualize many more optimum shapes for slender bodies of revolution at supersonic speeds, but later his idea of giving the trailing vortex sheet a finite thickness, of distinguishing between natural and artificial causes for singularities, permitted extending Von Kármán's theory to nonslender bodies (Ref. 2).

His well-known Busemann formula, which includes the centrifugal correction to the pressure on a body in hypersonic fiow, has served as the basis for many, now classical papers written by other authors on minimum drag bodies using variational techniques. Among them, we mention W. D. Hayes, A. Miele, G. G. Chernyi, A. L. Gonor, and J. Cole.

Dr. Busemann's active involvement in optimization problems took a new direction in 1965 when he presented the Ludwig Prandtl Memorial Lecture before the joint annual meeting of the German Association for Applied Mathematics and Mechanics (Ref. 3). His concept of the three-dimensional phase space (the Busemann space, in which all coplanar, Keplerian ellipses and hyperbolas are represented by points) has many interesting properties and allows a visualization of the reachable domain obtained by small variations from the given ellipse or hyperbola. By using Contensou's theory of convexization of this reachable domain resulting from an infinitesimal impulse, he obtained the optimal propagation in orbital transfer. In more familiar language, Busemann has shown the upper and the lower limits for the optimal thrust direction in the problem of free-time, coplanar orbital transfer. These limits form a useful angle for the optimal thrust direction and were studied analytically by Marchal, resulting in one of the most referenced papers in orbital impulsive transfer. The actual computation of these limits, as a function of the eccentricity and the true anomaly of the impulse on the transfer orbit, was carried out by R. D. Culp, one of Busemann's students. The results were published in this journal, thus making available to astrodynamicists one of the most useful, complete, and accurate set of data on the problem of orbital transfer. Details of the analysis, which provide accurate information for the sensitive problem of optimal guidance along a nearparabolic orbit, have been used in the preliminary design for the Viking mission to Mars. In 1967, Dr. Busemann was presented the Ludwig Prandtl Ring, an honor reserved for outstanding contributions to aeronautics, at the annual Prandtl Memorial Lecture. His continuing work on optimal trajectories, both in orbital flight and during reentry, was 
sponsored by many NASA grants and resulted in several NASA contractor reports and technical papers, some of them published in this journal.

A distinctive feature of Busemann's research is that his ideas are always stimulating for other people. Each of his publications, either in gasdynamics or in trajectory optimization, is always followed by a number of publications by younger scientists, who employ his approach with a kind of absolute conviction that they are following a leader in the field. They are correct, because Dr. Busemann always prepares his publications with care.

Over forty years ago, in Germany, Ludwig Prandtl was asked to write the article on Gasdynamics in the Handbuch der Physik. Since, at that time, he was about to finish his famous monograph on Hydrodynamics and Aerodynamics, he suggested the name of Jakob Ackeret. A few years later, on the same topic for the Handbuch der Experimental Physik, Prandtl suggested Busemann's name. On this occasion, Dr. Busemann wrote: "Such an opportunity to publish quickly and on request, at a time when all journals of applied sciences were overloaded with manuscripts, had to be used with caution when there was practically nothing new to report since the Ackeret article." His initial thought was that, since there was a lapse of only a few years between the two publications, the best that he could probably achieve was to generalize Ackeret's treatment for perfect gases with constant specific heats by some new approach potentially useful in analyzing general gases. This generalization evolved into the now famous Busemann shock polar and grew to the extent that the initially contracted size of 60 pages was increased to 120 pages. However, the proposed extension of Ackeret's treatment to analyzing unsteady gasdynamics had to be left out. Ten years later, during World War II, Dr. Busemann presented an updated version of the deleted portion on unsteady gasdynamics at a meeting on explosions and shaped charges of the German Academy of Aeronautics. At the same time, similar topics were treated in Russia by L. I. Sedov and in England by G. I. Taylor.

With the same care, Dr. Busemann has published nearly 100 technical papers. And with the same innovative view, he holds some patents in the aerospace field; but, since he is so far ahead in his field, his patents usually expire before they are put into commercial use.

It is with high regard for Dr. Busemann's achievements that this special issue of the Journal of Optimization Theory and Applications is dedicated in his honor. All of the contributed articles are invited papers. With real care the authors of this special publication have labored over their equations and perfected their methods. Collectively, they have 
produced a special issue of JOTA dealing with optimization problems in aerospace engineering and related problems as a testimony in honor of a man considered to be one of the most respected and outstanding scholars in aerospace engineering and applied mathematics.

\author{
N. X. Vinh \\ University of Michigan \\ Ann Arbor, Michigan
}

\title{
References
}

1. Busemann, A., Vortex Distributions of Minimum Energy, Oral presentation on the 25th Anniversary of Prandtl's First Wind Tunnel, Goettingen, Germany, 1933.

2. Busemann, A., About the Minimum Drag Nose, Physics of Fluids, Vol. 7, No. 8, 1964.

3. Busemann, A., Minimalprobleme der Luft- und Raumfahrt, 1965 Ludwig Prandtl Memorial Lecture, Vienna, Austria, 1965; Zeitschrift fur Flugwissenschaften, Vol. 13, pp. 401-411, 1965.

\section{Publications of Professor Adolf Busemann in the Area of Optimization Theory}

1. Nose Shapes for Supersonic Speeds, Schriften der Akademie der Luftfahrtforschung, 1945 (with G. Guderley).

2. Minimum Problems of Supersonic Flow, Lecture at the Academy Celebration of Prandtl's 70th Anniversary, Goettingen, Germany, 1945.

3. The Problem of Drag at High Subsonic Speeds, Journal of the Aeronautical Sciences, Vol. 16, pp. 337-344, 1949.

4. Minimum Virtual Mass, Jubilé Scientifique de M. Dimitri P. Riabouchinsky, P'ublications Scientifiques et Techniques du Ministère de l'Air, Paris, France, pp. 25-40, 1954.

5. The Relation Between Minimizing Drag and Noise at Supersonic Speeds, Proceedings of the Conference on High-Speed Aeronautics, Polytechnic Institute of Brooklyn, Brooklyn, New York, pp. 133-144, 1955.

6. About the Minimum Drag Nose, Physics of Fluids, Vol. 7, pp. 1386-1387, 1964. 
7. Minimal-Probleme der Luft- und Raumfahrt, Zeitschrift für Flugwissenschaften, Vol. 13, pp. 401-411, 1965.

8. An Approach to the Problem of Optimizing Orbital Maneuvers, Proceedings of the Mid-American Universities Association's Conference on Modern Optimization Techniques, Manhattan, Kansas, 1966 (with R. D. Culp).

9. Geometric Constraints of the Disorbit Problem, American Astronautical Society, Science and Technology Series, Vol. 11, pp. 309-402, 1967 (with N. X. Vinh and R. D. Culp).

10. Geometric Theory of Optimum Disorbit Problems, NASA Report No. CR-750, 1967 (with N. X. Vinh).

11. Sonic Boom Reduction (Contributed Remarks), Proceedings of the NASA Conference on Sonic Boom Research, NASA SP-147, pp. 79-82, 1967.

12. Optimum Constrained Disorbit by Multiple Impulses, Journal of Optimization Theory and Applications, Vol. 2, pp. 40-64, 1968 (with N. X. Vinh).

13. Optimum Maneuvers of Hypervelocity Vehicles, NASA Report No. CR-1078, 1968 (with N. X. Vinh and G. F. Kelley).

14. The Feasibility of Large Sonic Boom Reductions, Proceedings of the NASA Second Conference on Sonic Boom Research, NASA SP-180, pp. 125-128, 1968.

15. General Remarks on Sonic Boom, Proceedings of the NASA Second Conference on Sonic Boom Research, NASA SP-180, p. 193, 1968.

16. Optimum Maneuvers of a Skip Vehicle with Bounded Lift Constraints, Journal of Optimization Theory and Applications, Vol. 3, pp. 243-262, 1969 (with N. X. Vinh and G. F. Kelley).

17. Optimum Altitude for Coasting Flight of a Hypervelocity Vehicle, Journal of the Astronautical Sciences, Vol. 21, pp. 32-48, 1973 (with N. X. Vinh and R. D. Culp).

18. A Study of Very High Altitude Manewvers Using Aerodynamic Forces, USAF Aerospace Research Laboratory, Report No. ARL 74-0104, Vol. 1, 1974 (with R. D. Culp).

19. On the Effects of Aerodynamic Force and Continuous Thrust in Very High Altitude Maneuvers at Orbital Speeds, USAF Aerospace Research Laboratory, Report No. ARL 74-0104, Vol. 2, 1974 (with R. D. Culp and C. Y. Yang).

20. On the Use of Aerodynamic Forces to Effect Maneuvers of Orbiting Vehicles, USAF Aerospace Research Laboratory, Report No. ARL 74-0104, Vol. 3, 1974 (with R. D. Culp and C. Y. Yang).

21. Optimum Maneuvers of Hypervelocity Vehicles Using Thrust and/or Aerodynamic Force, USAF Aerospace Research Laboratory, Report No. ARL 740104, Vol. 4, 1974 (with N. X. Vinh and R. D. Culp). 
22. Optimum Three Dimensional Atmospheric Entry From the Analytical Solution of Chapman's Exact Equations, NASA Report No. CR-132571, 1975 (with N. X. Vinh and R. D. Culp).

23. Analytical Solution of the Optimal Three Dimensional Reentry Problem Using Chapman's Exact Equations, 25th Congress of the International Astronautical Federation, Paper No. 74-018, 1974 (with N. X. Vinh and R. D. Culp). 